\title{
Pourquoi et comment informer le patient sur les coûts
}

\author{
Valérie Junoda, Jean-Blaise Wasserfallen ${ }^{b}$ \\ a Professeure à la Faculté de droit de I'UNIGE et à la Faculté des HEC de I'UNIL; \\ b Vice-Directeur médical du CHUV, Professeur titulaire à la Faculté des HEC de I'UNIL
}

\begin{abstract}
Rares sont les professionnels de la santé qui renseignent sur le coût des prestations. Une lacune à combler vis-à-vis du patient sur lequel pèsent déjà de lourdes charges liées à la santé. Pour améliorer la transmission d'informations, des solutions impliquant tous les acteurs concernés sont à envisager.
\end{abstract}

En Suisse, une majorité des assurés a choisi une franchise supérieure au minimum de 300 francs. Plus de $20 \%$ ont opté pour la franchise maximum de 2500 francs [1]. Avec la quote-part plafonnée à 700 francs, ces personnes s'exposent à payer de leur poche 3200 francs par an, voire plus en cas de soins stationnaires. En dépit de cette lourde charge financière, les professionnels de la santé se désintéressent encore largement du coût des prestations incombant à leurs patients. Une attitude surprenante, car pour la plupart des autres prestations de service, les tarifs sont soit publics, soit renseignés.

Le Tribunal fédéral, dans un arrêt datant de 1993 (ATF 119 II 456), avait rappelé que les professionnels de la santé sont tenus, vis-à-vis de leurs clients, à un devoir «minimal» d'informations sur les conséquences financières des traitements. Il se prononçait dans un cas où la prestation n'était pas - ou peut-être pas - couverte par l'assurance-maladie de base. Le patient avait donc besoin de savoir à quelles conditions la prestation serait prise en charge, et à supposer ces conditions non satisfaites, le coût approximatif qu'il devrait assumer. Le non-respect de l'obligation de renseigner ne rend pas le traitement illicite, mais expose le soignant à en assumer le coût. De même, le Code de déontologie de la FMH exige en cas de «doute quant à la prise en charge du traitement par l'assurance du patient, [que] le médecin en informe celui-ci et lui demande de vérifier la question du remboursement auprès de son assureur» (art. 11). De manière surprenante, le Code met la vérification à la charge du patient, le médecin n'en étant pas responsable.

Même si la jurisprudence et le Code font référence au cas d'une prestation hors assurance obligatoire des soins (AOS), la situation n'est guère différente, du point de vue du patient, lorsque le coût lui incombe en raison d'une franchise élevée et non atteinte. En pratique, le deuxième cas de figure est plus fréquent que le premier. Les soignants devraient donc mieux renseigner sur le coût des prestations, tout particulièrement lorsque le médecin traitant réfère son patient à un spécialiste ou à l'hôpital, le patient pouvant encore moins bien prévoir les coûts engendrés.

\section{Que peuvent faire les médecins?}

Pour les médecins, il n'est pas aisé de mettre en œuvre une obligation d'informer sur les coûts. Ils ne savent souvent pas précisément combien les prestations prescrites coûtent. Dans le domaine hospitalier stationnaire (SwissDRG), les coûts sont difficiles à prévoir (ex ante) puisqu'ils dépendent de la combinaison de codes diagnostiques et opératoires arrêtés seulement en fin de séjour. En ambulatoire, les coûts résultant du TARMED demeurent difficiles à anticiper pour le soignant. Pour le patient, tant le TARMED que le SwissDRG sont peu compréhensibles en raison des milliers de positions recensées.

La réticence des médecins à informer peut aussi s'expliquer par le souci de ne pas donner l'impression que les choix médicaux sont dictés par les coûts. Le médecin qui recommanderait à son patient «de faire ceci, mais attention, cela pourrait vous coûter tant» envoie un message potentiellement contradictoire. Si le prix est élevé, le patient pourrait penser que le médecin le dissuade en réalité d'entreprendre la mesure pourtant recommandée ou qu'il entend le culpabiliser sur les coûts engendrés. Si le prix est au contraire bas, le pa- 
tient pourrait penser que le médecin lui recommande une médecine au rabais par souci d'économie. Même si l'information du médecin est accompagnée d'un rapport coût-bénéfice, elle pourrait susciter l'anxiété chez certains patients. Une personne gravement malade n'est souvent pas à même d'analyser et d'interpréter un tel rapport, encore moins d'opérer elle-même la sélection des actions qui en résultent; c'est la conviction que le médecin met tout en œuvre pour la soigner au mieux, indépendamment des coûts, qui la rassure.

Ces réticences sont compréhensibles lorsque les pouvoirs publics et les assureurs sont les seuls à décider des prestations à rembourser. Elles le sont moins lorsque le patient doit lui-même payer les coûts. Cette situation vise surtout des assurés plutôt jeunes et en bonne santé, avec une franchise élevée, ou des assurés ayant besoin de prestations qui ne sont pas à charge de l'AOS (p. ex. soins dentaires). Ces motifs notamment amènent 10 à 20\% de la population [2] à renoncer à des soins. Dès lors, il serait préférable que ceux qui font face à de telles décisions puissent le faire en connaissant à la fois le coût et le bénéfice de la mesure à entreprendre ou à sacrifier. Tous les patients n'osant pas demander à leur médecin si la mesure qu'il préconise «en vaut la chandelle au vu du coût», l'initiative doit venir des professionnels de la santé.

\section{On constate des progrès en matière d'informa- tion de la part des professionnels de la santé.}

Sur ce plan, des progrès ont été constatés. Améliorer la qualité des soins et celle de l'information fournie aux patients est un mouvement en plein essor. Des directives en tout genre aident à choisir les bonnes approches et à renoncer aux prestations inutiles sur la base d'un rapport bénéfice-risque, sans connaissance nécessaire des coûts impliqués (p. ex. Choosing Wisely, Top 5 Smarter Medicine). En parallèle, les professionnels de la santé s'efforcent d'élaborer des fiches d'information compréhensibles pour les patients. Toutefois, cette tendance est encore trop éloignée des préoccupations pécuniaires. Lorsqu'une thérapie, une maladie ou un traitement est expliqué aux médecins, respectivement aux patients, les explications scientifiques fournies sont rarement accompagnées de chiffres précis sur les coûts.

Pourtant, même pour le médecin, une information sur les coûts peut faciliter sa prise de décision. Les pressions exercées par les caisses effectuant les contrôles d'économicité devraient inciter les médecins à prendre en compte cette variable dans leur choix, subsidiairement au rapport bénéfice-risque.

\section{Que peuvent faire les caisses maladie?}

Les caisses maladie et leurs associations faîtières pourraient établir des brochures expliquant les coûts attendus pour les prestations médicales les plus courantes. Le médecin remettrait alors à son patient cette brochure comme support, voire base de discussion dans les cas plus complexes. Idéalement, le dépliant devrait aussi évoquer les bénéfices de la prestation. Le médecin ne le fait souvent que succinctement: «la radiographie permet de confirmer le diagnostic»; «la physiothérapie aide à la réhabilitation"; "l'analyse de sang peut détecter une déficience en fer». Des explications certes logiques et convaincantes pour le patient, mais ne disant rien sur l'ampleur du bénéfice par rapport à son coût. Si l'information sur les coûts probables est difficile à standardiser, celle sur le rapport bénéficecoût l'est encore davantage, sans qu'il faille pour autant y renoncer.

Une autre piste porte sur les modalités de la facturation. Favorisant le système dit «tiers garant», les caisses se plaisent à répéter qu'il incombe aux assurés de contrôler les factures reçues. Cependant, le pourcentage d'assurés signalant à leurs caisses des erreurs est probablement très faible. Si les caisses veulent sensibiliser les assurés aux coûts et les inciter à vérifier leurs factures, elles devraient en préparer une version simplifiée.

Chaque caisse devrait de surcroît envoyer à chaque assuré un résumé annuel sur un modèle standardisé. Cette information synthétique fournirait aux assurés une vue d'ensemble de leur parcours médical. Ce document annuel faciliterait aussi le suivi et la coordination des soins. Les outils d'intelligence artificielle qui se développent devraient permettre d'analyser les données brutes des caisses et d'offrir un tel service pour un coût raisonnable.

Finalement, il serait utile d'inclure les factures et le récapitulatif annuel susmentionné dans le dossier électronique du patient (DEP) pour garder une trace des dates de traitement et des professionnels consultés. Ceci n'est toutefois pas prévu actuellement. Le DEP appartenant au patient, et non à l'assureur [3], lui permettre de consulter toutes ses factures adressées à sa caisse sur un site unique constituerait un atout. Le suivi longitudinal et la vue d'ensemble en seraient aussi facilités, pour le médecin traitant aussi. Tant que le DEP ne sera pas utilisé dans tous les cabinets, il est intéressant de compléter cette base de données avec les factures en mains des caisses. Le patient peut déjà inclure cette information lui-même dans certains DEP, ce qui est toutefois fastidieux. 


\section{Que peuvent faire les revues médicales?}

Alors que les articles médicaux, notamment ceux publiés dans le BMS et d'autres revues suisses, détaillent méticuleusement les bénéfices et risques des traitements, leurs coûts, ne serait-ce qu'approximatifs, sont généralement omis. Pourtant, cette information aide à analyser la pertinence pratique du traitement. Même si une information exhaustive est impossible à fournir, vu les variations possibles notamment en cas de complications, l'indication d'un tarif de base ou d'un tarif moyen serait précieuse. En renseignant sur les coûts, les journaux contribueraient à instaurer chez le médecin un réflexe de s'informer, puis d'informer à son tour ses patients.

Les revues pourraient aussi aborder le thème des coûts via des vignettes ou des études de cas. Bien que compliqués, les aspects liés à la facturation ne sont pourtant pas considérés comme suffisamment "scientifiques" pour un article médical. Par exemple, les différences de coûts entre certaines interventions en ambulatoire ou en stationnaire peuvent être significatives: elles sont toutefois discutées presque uniquement sous un angle politique, alors qu'une discussion scientifique et professionnelle se justifie pleinement.

Valérie Junod

Professeure associée UNIL HEC

Professeure titulaire UNIGE Droit Bureau MAIL 4081

Faculté de droit Boulevard du Pont-d'Arve 40 CH-1211 Genève 4 valerie.junod[at]unil.ch

\section{Rassurer le patient en l'informant}

De nos jours, le randonneur qui engage un guide de montagne pour son excursion, le parent qui engage un répétiteur pour son enfant, le contribuable qui confie l'établissement de sa déclaration d'impôts à une fiduciaire savent combien ils vont payer et ont une idée assez précise du bénéfice escompté. Dans le secteur médical, trop souvent, le patient est dans le flou. La technicité de la science médicale n'explique qu'en partie cette situation. Notre société prône toujours davantage l'autodétermination et la responsabilisation individuelle des patients. Cependant, elle ne leur donne pas toujours les moyens d'y parvenir. Vouloir imposer une responsabilité financière plus grande, comme il en a été question lors des débats récents sur les franchises, sans fournir la formation et les outils adéquats est simplement irréaliste, voire injuste. D’abord, le patient doit être en mesure de faire des choix éclairés. C'est seulement ensuite qu'il pourrait éventuellement être tenu responsable des coûts qui en découlent. En tout état de cause, la responsabilité devrait être assumée en première ligne par les soignants et par un système qui fournit les bonnes incitations. On entend souvent dire que les patients mettent les médecins sous pression en exigeant toujours davantage de prestations, mais on oublie qu'un patient inquiet doit d'abord être rassuré, informé, guidé et accompagné dans ce processus. Sinon, les espoirs mis dans une responsabilité individuelle, en quelque sorte «innée», resteront vains.

\section{Références}

1 Conseil fédéral, Participation aux coûts dans l'assurance obligatoire des soins, Rapport du Conseil fédéral en réponse au postulat Schmid-Federer du 22.3.2013 (13.3250 «Effets de la franchise sur la consommation de prestations médicales»), 28.6.2017.

2 ASSM, Le rationnement au sein du système de santé suisse: analyse et recommandations, 2007; Rapport du groupe d'experts, Mesures visant à freiner la hausse des coûts dans l'assurance obligatoire des soins, août 2017.

3 Il n'est bien sûr pas question de donner aux assureurs accès au DEP; ils seraient uniquement tenus d'y «uploader» les factures. Via les factures, les caisses ont de toute façon déjà une connaissance assez précise de l'état de santé de leurs assurés.

\section{L'essentiel en bref}

- Les professionnels de la santé renseignent trop peu le patient sur le coût des traitements, malgré l'importance de cette information notamment pour les assurés dont la franchise est élevée et non atteinte.

- Si le patient reçoit de plus en plus de fiches informatives sur une maladie et son traitement, rares sont celles contenant des chiffres sur les coûts. Les médecins eux-mêmes sont mal informés, alors même que ces indications les aideraient dans leur prise de décision.

- Les caisses maladie pourraient établir une liste des coûts à prévoir pour les prestations médicales courantes. Le médecin remettrait cette liste à son patient. Les caisses devraient aussi envoyer aux assurés des factures simplifiées ainsi qu'un résumé annuel.

- Les revues médicales devraient davantage aborder les aspects liés à la facturation. Cela inciterait le médecin à s'informer, puis à informer à son tour ses patients.

\section{Das Wichtigste in Kürze}

- Die Gesundheitsberufe klären den Patienten zu wenig über die Behandlungskosten auf, obwohl diese Information vor allem auch für Versicherte mit hoher und nicht in Anspruch genommener Franchise von Bedeutung ist.

- Der Patient erhält zwar immer mehr Informationsblätter zu Krankheiten und zu deren Behandlung, doch selten sind darauf auch die entsprechenden Kosten abgebildet. Auch die Ärzte selbst sind schlecht informiert, wobei doch gerade diese Angaben Hilfestellung bei der Entscheidung leisten könnten.

- Die Kassen könnten eine Kostenliste für die anfallenden medizinischen Leistungen erstellen. Der Arzt würde diese Liste an den Patienten weiterreichen. Die Kassen sollten dem Versicherten auch vereinfachte Rechnungen und eine Jahresrechnung zukommen lassen.

- Medizinische Fachzeitschriften sollten vermehrt auf die Rechnungsstellungsthematik eingehen. So könnte der Arzt zuerst sich selbst und in der Folge auch seine Patienten informieren. 